\title{
Oh, the places we'll go! Emergency department extracorporeal cardiopulmonary resuscitation (ECPR) in Canada
}

\author{
James B. Gould, MD*; Paul Atkinson, MB, MA ${ }^{\dagger}$; George Kovacs, MD, MHPE*
}

The narrative of any new innovation in medicine is that of a journey met with challenges and barriers. In their recent report from the first meeting of the Canadian ECPR Research Working Group, Brooks et al. identify those barriers for extracorporeal cardiopulmonary resuscitation (ECPR) and provide a framework of opportunity going forward within the Canadian context. ${ }^{1}$

There are over 40,000 cardiac arrests in Canada every year, with $85 \%$ of those occurring out of the hospital. Although survival rates have improved over time, out-of-hospital cardiac arrests treated by emergency medical services have favourable outcomes in less than $10 \%$ of cases. $^{2}$ Furthermore, patients without a sustained return of spontaneous circulation within the first 30 minutes of their resuscitation, termed refractory arrest, have exceptionally lower rates of good neurological outcome. ${ }^{3}$ Fortunately, observational data have shown survival rates with good neurological outcome as high as $30 \%$ to $40 \%$ for refractory cardiac arrest when using ECPR. ${ }^{3-4}$

In their report, Brooks et al. identify six high priority questions related to ECPR research and program implementation. Perhaps most importantly, they ask, "What are the best practices in ECPR to optimize neurologically favourable survival?" Because this endeavour is crucial, we must not forget that ECPR programs must also coexist within their local system. Health systems are complex. Their differences will result in a variability of effect and response to what we, in the controlled research setting, have determined to be best practice. As such, in the development of an ECPR program, we must either determine a practice that best accommodates our local system or otherwise change the system to accommodate the best practice, whatever that may be.

While implementing ECPR research in Canada, we need to prepare ourselves for the results of that research. Both health professionals and the public need to rethink what constitutes a successful outcome in cardiac arrest. Although a neurologically favourable outcome is important and is certainly the primary objective of any ECPR program, it is not the only success of the intervention. Failure at the level of individual survival may still provide societal benefit through organ donation programs. ${ }^{5}$ Organ donation has been shown to be a positive experience for families in terms of closure, by contribution to the lives of others. ${ }^{6}$ Perhaps we need to consider that a success $-\mathrm{a}$ benefit, in any cost-benefit analysis. Perhaps we need to reflect this outcome in research protocols. The question then becomes, How do we capture the success in the patient and family experience? This likely requires a call for inclusion of qualitative study in ECPR research and a call for more inclusion of patients and families on both study teams and working groups.

As we begin to implement programs, we need to anticipate failures and understand that they are acceptable not only because the alternative for these patients is assured death, but also because failure can provide an opportunity for improvement. Building a malleable ECPR program might be the most important component of its design. Programs need to have the ability to evolve with time, adapt to change, and respond to these failures. To accommodate what we determine to be best practice, there is no point in building a program that does not have the capacity to evolve when we inevitably redefine best

From the *Department of Emergency Medicine, Dalhousie University, Halifax, NS; and tDepartment of Emergency Medicine, Dalhousie University, Saint John, NB.

Correspondence to: Dr. James B. Gould, 1796 Summer Street, Halifax Infirmary, Suite 355, Halifax, NS B3H 3A7; Email: james.gould@ dal.ca 
practice. This requires continuous rigorous quality improvement strategies and research methods that can prospectively identify potential failures before they occur and make suggestions to avoid them. ${ }^{7}$ The goal should be to set up for success but prepare how to deal with failure. If the program fails, then hopefully it fails fast, so we can fix it faster.

Finally, as noted by the authors, there needs to be clarity, along with a research base, for the emergency physicians' (EP) roles in ECPR. Three areas of focus are the point-of-care ultrasound (POCUS), cannulation, and team leadership. The ability of EPs to use POCUS to identify key reversible causes and to identify and guide vascular access may be unique in certain settings. ${ }^{8}$ To suggest that EPs could be involved in vascular access and cannulation is not meant to be self-serving or to suggest that ECPR should occur without cardiovascular surgery involvement. Rather it is meant to expedite the multidisciplinary process to improve time to ECPR and subsequently patient outcomes. We are comfortable with femoral access, we have historically used femoral vessel cannulation in resuscitation, and we have been amongst the first to advocate for ultrasound use in that context. ${ }^{9}$ Furthermore, EPs will undoubtedly be the first physicians available to these patients and thus have an opportunity to initiate the process. Fortunately, this would not be the first time that our specialty has encountered an expansion in our scope of practice in resuscitation. Not unlike endotracheal intubation or resuscitative thoracotomy, vascular access and cannulation in ECPR is a high acuity, low opportunity scenario. Similar to how we obtained excellence in those procedures, we need to develop training programs for POCUS and vascular access and cannulation in the simulated environment with vascular models and clinical cadavers. In doing so, we need to engage our surgical colleagues and benefit from their expertise as educators in those training programs. ${ }^{7}$

As emergency medicine specialists, we define ourselves by our ability to resuscitate patients. The primary purpose of the EP is to be there to save lives; while we are waiting for our patients, we also try to help anyone else who shows up. Our emergency department (ED) teams specialize in their ability to create a sense of calmness out of what is otherwise chaos. With the addition of many new team members and a new complex procedure, ECPR activations in the ED have the potential to create chaos. Thus, these ECPR cases will require advanced leadership skills, with excellent crisis resource management. Who better than the EP, a specialist in chaos, to lead that team.

As the narrative of ECPR in Canada unfolds, we must consider the associated barriers and opportunities in research and program development. While we embark on that journey, we must consider how to determine best practice, how to define success, and how to best use the expertise of the EP. "We're off to great places, today is our day, our mountain is waiting, so, let's get on our way!"10

Keywords: cardiac arrest, extracorporeal cardiopulmonary resuscitation, extracorporeal membrane oxygenation

Competing interests: None declared.

\section{REFERENCES}

1. Brooks SC, Shemie SD, Torrance S, et al. Barriers and opportunities related to extracorporeal cardiopulmonary resuscitation for out-of-hospital cardiac arrest in Canada: a report from the first meeting of the Canadian ECPR Research Working Group. CFEM 2018;20(4):507-17.

2. Daya MR, Schmicker RH, Zive DM, et al. Out-of-hospital cardiac arrest survival improving over time: results from the Resuscitation Outcomes Consortium (ROC). Resuscitation 2015;91:108-15.

3. Matsuyama T, Kitamura T, Kiyohara K, et al. Impact of cardiopulmonary resuscitation duration on neurologically favourable outcome after out-of-hospital cardiac arrest: a population-based study in Japan. Resuscitation 2017;113:1-7, doi:10.1016/j.resuscitation.2017.01.005.

4. Yannopoulos D, Bartos JA, Raveendran G, et al. Coronary artery disease in patients with out-of-hospital refractory ventricular fibrillation cardiac arrest. $7 \mathrm{Am}$ Coll Cardiol 2017;70(9):1109-17, doi:10.1016/j.jacc.2017.06.059.

5. Casadio MC, Coppo A, Vargiolu A, et al. Organ donation in cardiac arrest patients treated with extracorporeal CPR: a single centre observational study. Resuscitation 2017;118:133-9.

6. Ralph A, Chapman JR, Gillis J, et al. Family perspectives on deceased organ donation: thematic synthesis of qualitative studies. Am $\mathcal{F}$ Transplant 2014;14:923-35.

7. DeRosier J, Stalhandske E, Bagian JP, Nudell T. Using healthcare failure mode and effect analysis: the VA National Center for Patient Safety's Prospective Risk Analysis System. 7 Qual Improv 2002;27(5):248-67.

8. Atkinson PR. Emergency department ECMO and echo better together? In response to: Soar J. An observational study of extracorporeal CPR for in-hospital cardiac arrest secondary to myocardial infarction. Emerg Med 7 2014;31:440.

9. Hilty WM, Hudson PA, Levitt MA, et al. Real-time ultrasound-guided femoral vein catheterization during cardiopulmonary resuscitation. Ann Emerg Med 1997;29:331-6.

10. Seuss Dr. Ob, the places you'll go! New York: Random House; 1990. 